\title{
A COMPACT PROTON SYNCHROTRON WITH COMBINED-FUNCTION LATTICE DEDICATED FOR CANCER THERAPY*
}

\author{
$\underline{\text { A. MORITA }}^{\dagger}$, A. NODA, M. INOUE, T. SHIRAI, and Y.IWASHITA ICR, Kyoto University, \\ K. HIRAMOTO, M. KATANE, M. TADOKORO, M. NISHI, \\ and M. UMEZAWA HITACHI Co. Ltd.
}

\section{Abstract}

A compact proton synchrotron with combined function lattice has been designed as a dedicated machine for cancer therapy because of its merits of easy operation and low construction cost. The lattice has a six-fold symmetry and its radius of curvature and circumference are $1.9 \mathrm{~m}$ and $23.9 \mathrm{~m}$, respectively. For the purpose of establishing a good reference design, we have constructed a model magnet based on the three-dimensional magnetic field calculation. Magnetic field measurement has been performed with use of three-dimensional Hall-probe. In the present paper, the results of these development is to be presented together with the outline of the reference design.

\section{INTRODUCTION}

Recently radiation cancer therapy has been paid attention because of its merit of preserving function and shape of human body and rather light load to the patient compared with the other therapy. Among radiation therapies, charged particle therapy has such a merit that it can localize the dose distribution to tumor largely reducing the damage to normal cells. In order to cure a lot of patients, it is required to construct at least one such facility in each prefecture, however, constructing and operating such charged particle therapy facility need many cost. From this point of view, We have been developing a compact proton synchrotron facility, which is easy for daily operation and required cost is rather limited[1]. As the machine for such proton accelerator, we have chosen a proton synchrotron with combined function lattice, because of its non-flexibility. This nonflexibility cause difficulty of tuning, however, such nonflexibility gives easy operation once a good design has been established. We develop the reference design of the combined function magnet with the help of three-dimensional magnetic field calculation code. In the present paper, we present our magnet system design and the measurement results of the model magnet.

\footnotetext{
* Work supported by Grant-in-Aid for Scientific Research from Ministry of Education, Science, Sports and Culture of Japan under the contact number 08559010

$\dagger$ morita@kyticr.kuicr.kyoto-u.ac.jp
}

\section{DEVELOPMENT PROCESS OF REFERENCE MODEL}

\subsection{Primary Design}

In our primary design, the synchrotron ring has a six-fold symmetry and the lattice is constructed by 60 degree bending sector magnet and 2 meters drift-space. On the central orbit in a bending magnet, the radius of curvature and the maximum field strength are $1.9 \mathrm{~m}$ and $1.28 \mathrm{~T}$, respectively to accelerate a proton up to $250 \mathrm{MeV}$. The focusing elements are implemented in bending magnets as FDF structure combined function[2]. For convenience of calculation, bending angles of F,D, and F sector are designed 15,30, and 15 degree, respectively. To set operating point $\nu \sim 1.75$, $\mathrm{n}$-value of magnet sector is given 6.164 for D-sector and -5.855 for F-sector by matrix calculation. We consider that the magnet is made by laminated iron plates. Thus, both transition region between $\mathrm{F}$ and $\mathrm{D}$ sectors and end edge regions are designed as step function to approximate potential made by Rogowski curved pole at the medium plane of the magnet. The structure of the synchrotron ring is shown in Fig.1.

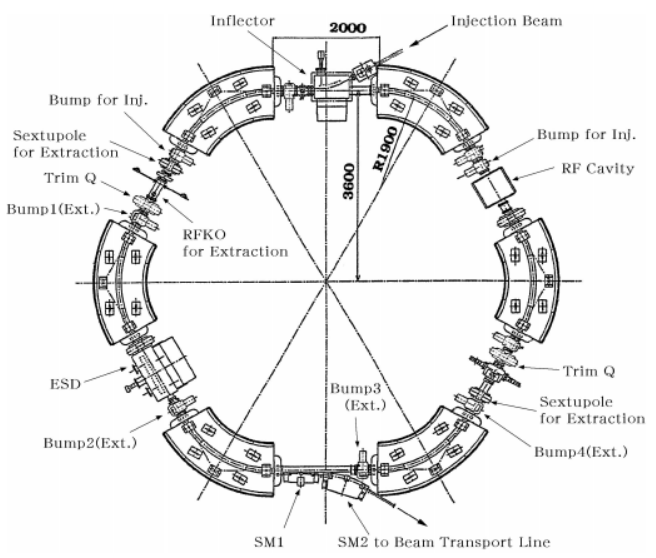

Figure 1: Structure of the synchrotron ring

\subsection{Operating Point Evaluation}

To verify the design, we evaluate the operating point. Both tune value and beta function are calculated using transfer matrix reconstruction method with particle tracking[3] on the magnetic field calculated by the three-dimensional magnetic field calculation code TOSCA. In the treatment of both fringing and transition of between $\mathrm{F}$ and $\mathrm{D}$ sectors 
and central-orbit deformation, this method based on particle tracking has advantage compared with ordinary method using the product of the transfer matrices defined by analytical results of uniform field.

\subsection{Operating Point Tuning}

The tune value evaluated from our primary design $\left(\nu_{h}, \nu_{v}\right) \sim(1.64,1.86)$ is far from the design target. To move operating point to the design target, we modify the ratio of bending angles, which increases bending angle of F-sector in $\triangle \theta$ degree and decreases bending angle of Dsector in $2 \triangle \theta$ degree. Of cause, for modifying tune value, $\mathrm{n}$-value modification is essential, however, the pole shape has already been determined with the two-dimensional field calculation using POISSON. Changing the ratio of bending angles seems to correspond to local n-value modification at the boundary between $\mathrm{F}$ and $\mathrm{D}$ sectors. Thus, the theory of perturbation predicts that the ratio of horizontal and vertical tune value modulation is given by ratio of beta function at the boundary and the modulation magnitude of tune value is proportional to the modulation of bending angle $\triangle \theta$. Using $\triangle \theta$ tuning, we can control tune value on the linear line.

\subsection{Model Magnet}

Considering the operating point and resonance lines, we select $\triangle \theta$ parameter to 0.25 degrees and operating point $(1.7,1.75)$ as the final design to avoid resonance lines up to 6 th order. To verify the reality of the three-dimensional calculation, we made a model magnet of the final design and made field measurement. Figure 2 shows the lower half

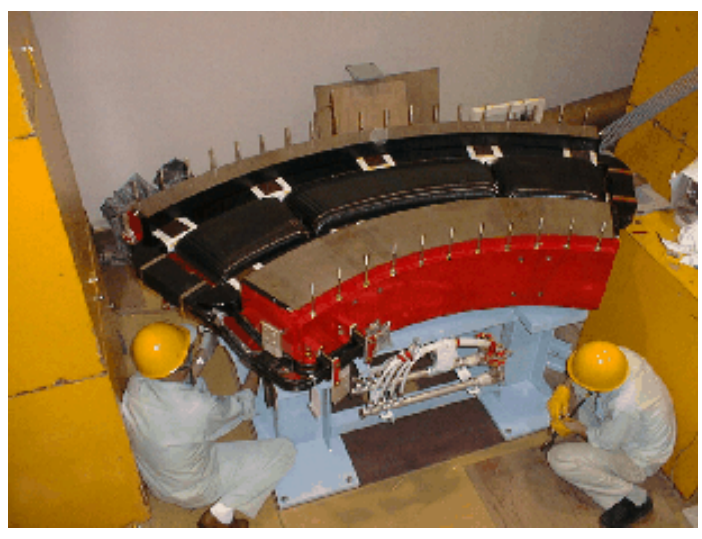

Figure 2: Lower half of the model magnet

of the completed model magnet. The poles of the magnet are made by the laminated iron plates whose thickness are $0.5 \mathrm{~mm}$

\section{MEASUREMENT OF MODEL MAGNET}

Considering the symmetry of the lattice structure, we measured the half of the magnet by mapping on the cylindrical coordinate using the three-dimensional Hall-probe ${ }^{1}$ fixed to the three-axis movable stage. The measurement has been performed by $5 \mathrm{~mm}$ spacing in radial direction from $1.84 \mathrm{~m}$ to $2 \mathrm{~m}$ and 1 degree spacing in circumferential direction from the center of $\mathrm{D}$ sector to out side of magnet with 43 points. These measurement setup and the Hall-probe structure are shown in Fig.3 and 4, respectively. From the

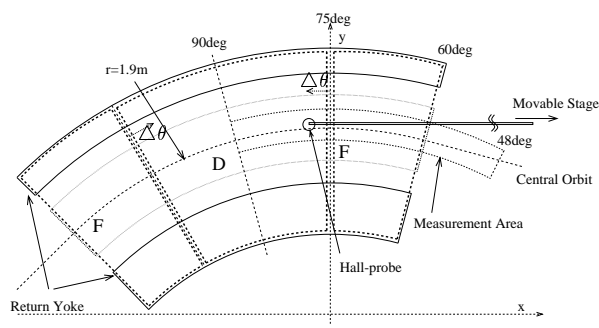

Figure 3: Geometry of the measurement

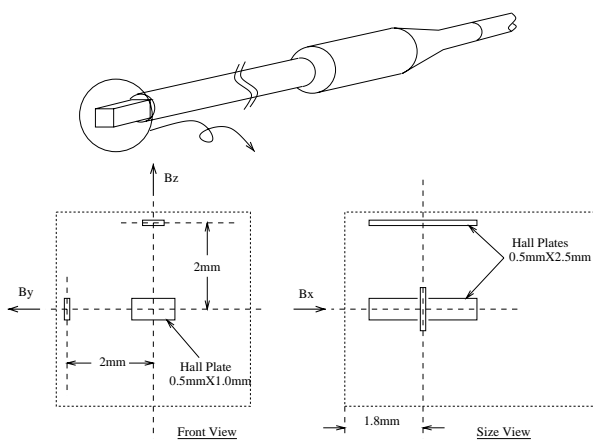

Figure 4: Structure of the Hall-probe

mirror symmetry between upper and lower poles, the magnetic field flux cross perpendicular to the medium plain. Thus the horizontal components of magnetic field should be equal to zero on the medium plane and anti-symmetric for the medium plane. But measured horizontal components shown in Fig.5 do not satisfy such symmetry and has obvious offset on the medium plane. These offset on the
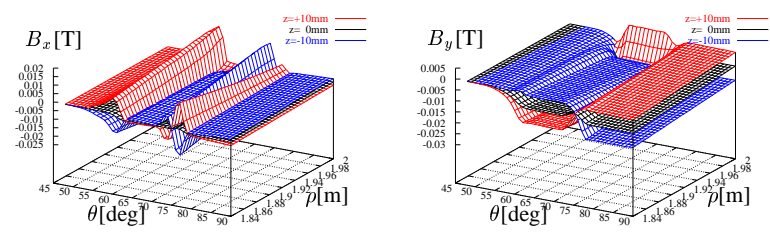

Figure 5: Raw horizontal components of the magnetic field

medium plane seems to be caused by vertical component $B_{z}$ shown in Fig.6. Thus we consider that these asymmetries are caused by the rotation of the probe axes, because of both bending and rotating probe arm. To correct those asymmetry, we introduce two parameters of rotating angle

\footnotetext{
${ }^{1}$ Lake Shore Model 460 \& MMZ-2500-UH
} 


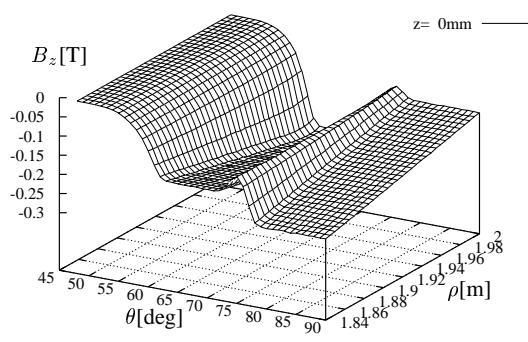

Figure 6: raw vertical component of the magnetic field

and determines these two parameters by fitting to minimize the ratio of square expectation value between horizontal components and vertical component. One of the corrected horizontal components are shown in Fig.7 and it is seem that symmetry is recovered.
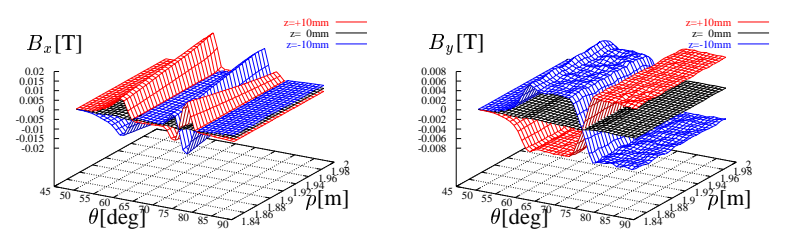

Figure 7: Corrected horizontal components of the magnetic field

\section{RESULTS OF MEASUREMENT}

To evaluate the magnetic field, we compared vertical components $B_{z}$ and n-value on the central orbit. The global feature $B_{z}$ and n-value calculated on the ideal central orbits ${ }^{2}$ shown in Fig. 8 and 9 have good agreement between measurement and TOSCA calculated in edge shape at fringing and F-D transition, respectively. But the magnitude of nvalue is slightly different in detail.

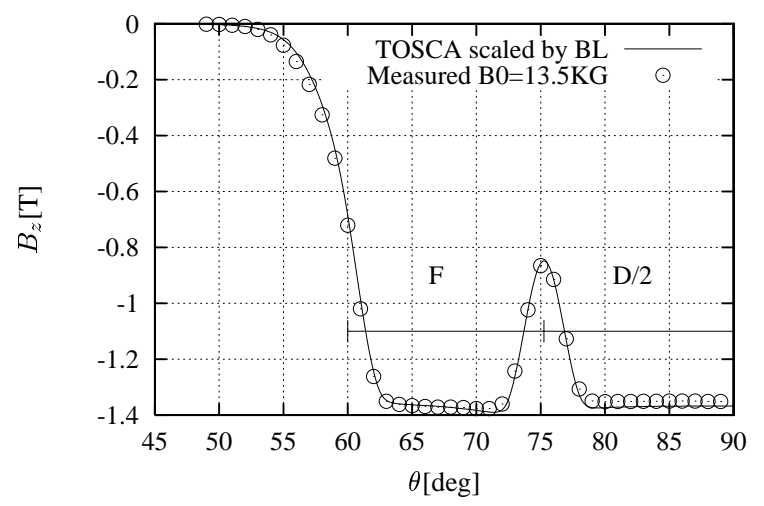

Figure 8: $B_{z}$ on the central orbit
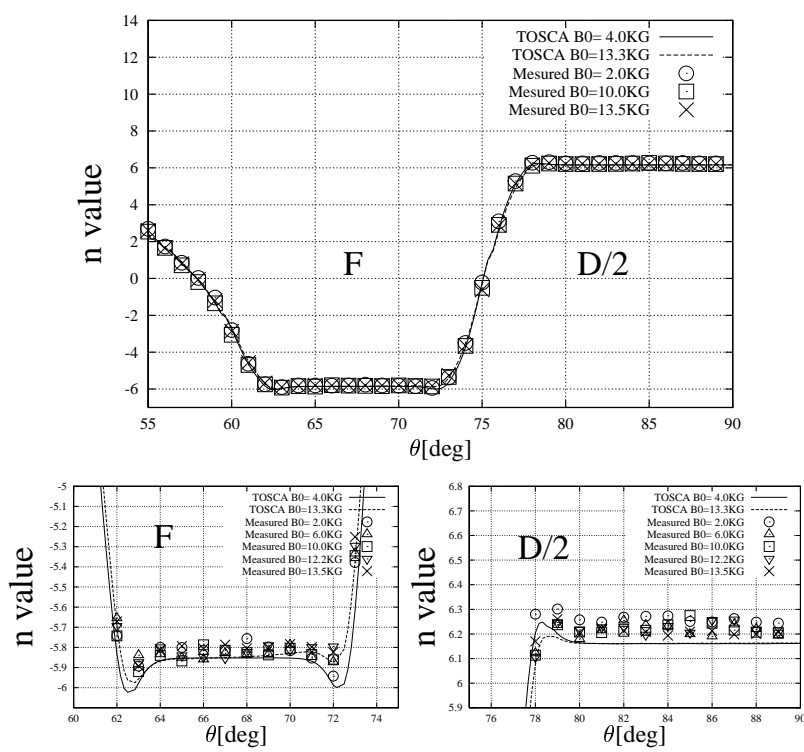

Figure 9: n-value on the central orbit

Following facts are considered as the problem in our nvalue measurement process. In calculating n-value from measured field, we use linear fit to 25 data points in linear region to calculate field gradient. Stable flat top of n-value in the center of each sectors is predicted from TOSCA results, however, measured n-value contain some deviations. Our gauss meter give us 5-digit as measurement result and field difference between adjusted measurement grids is the order of 1 percent of the field strength. Then, the measurement precession is not enough to discuss n-value and tune value calculation on these field map.

To treat the magnitude of n-value more in detail, improvement of measurement precision by direct measurement of field gradient is now under investigation.

\section{ACKNOWLEDGEMENT}

The authors would like to present their sincere thanks to Drs. H. Ogawa, K. Noda and M. Kumada at NIRS for their support during three-dimensional field measurement.

\section{REFERENCES}

[1] A. Noda et al., "Dedicated accelerator project for proton therapy at Kyoto University", NIRS-M-103, HIMAC-008, Proc. of the NIRS International Seminar on the Application og Heavy Ion Accelerator to Radiation Therapy of Cancer in connection with the 21th PTCOG Meeting (1994) 292-294.

[2] M. Tadokoro et al., "A combined Function Magnet for a Compact synchrotron", Proc. of the 1997 Particle Accelerator Conference, Vancouver, Canada (1997) 3294-3296.

[3] A. Morita et al., "Tune Value Evaluation for Combind Dunction Lattice", Beam Science and Technology, Vol 3(1998) 2326.

\footnotetext{
${ }^{2} 1.9 \mathrm{~m}$ curvature radius on the medium plane
} 